\title{
The Connectivity of the Basis Graph of a Branching Greedoid
}

\author{
Li Xueliang* \\ DEPARTMENT OF APPLIED MATHEMATICS \\ UNIVERSITY OF TWENTE \\ 7500 AE ENSCHEDE, THE NETHERLANDS
}

H. J. Broersma

\section{ABSTRACT}

A result of Korte and Lovász states that the basis graph of every 2connected greedoid is connected. We prove that the basis graph of every 3 -connected branching greedoid is $(\delta-1)$-connected, where $\delta$ is the minimum in-degree (disregarding the root) of the underlying rooted directed (multi)graph. We also give examples showing that this result is (in some sense) best possible.

\section{INTRODUCTION}

A result of Korte and Lovász [2] states that the basis graph of every 2connected greedoid is connected. This also implies that every spanning tree of a 2-connected rooted graph can be changed into a given second one by successively exchanging a single leaf in every step. As part of a general study on the diameter of basis graphs for different classes of greedoids, Ziegler [3] showed that the number of exchanges of leaves in the above procedure can be bounded above by $r^{2}-r+1$, where $r$ is the rank of the greedoid. A natural problem is whether there are a number of completely different (independent) ways to change one spanning tree into another. This problem asks for information on the connectivity of the basis graph. In this paper we prove that the basis graph of a 3-connected branching greedoid is $(\delta-1)$-connected, where $\delta$ is the minimum in-degree (disregarding the root) of the underlying rooted directed (multi)graph. We give examples to show that this lower bound cannot be improved.

*On leave from the Department of Mathematics, Xinjiang University, Urumchi, Xinjiang. Peoples Republic China.

Journal of Graph Theory, Vol. 16, No. 3, 233-237 (1992)

(c) 1992 John Wiley \& Sons, Inc.

CCC 0364-9024/92/030233-05\$04.00 


\section{TERMINOLOGY}

We refer to [1] for terminology and results on greedoids, but repeat the definitions for the notions we use in this paper.

A greedoid on a finite set $E$ is a pair $(E, \mathscr{F})$, where $\mathscr{F}$ is a nonempty collection of subsets of $E$ satisfying:

(1) $\phi \in \mathscr{F}$.

(2) For every nonempty $X \in \mathscr{F}$ there is an $x \in X$ such that $X \backslash\{x\} \in \mathscr{F}$.

(3) For $X, Y \in \mathscr{F}$ such that $|X|>|Y|$, there is an $x \in X \backslash Y$ such that $Y \cup\{x\} \in \mathscr{F}$.

The sets in $\mathscr{F}$ are called feasible. Thus a greedoid is a matroid if and only if every subset of a feasible set is again feasible. The maximal feasible sets of a greedoid are called its bases. Like in the case of matroids, (3) implies that all bases of a greedoid have the same cardinality, which we call the rank of the greedoid. Two bases $X$ and $Y$ of a greedoid $(E, \mathscr{F})$ are called adjacent if $|X \cap Y|=|X|-1$ and $X \cap Y \in \mathscr{F}$, that is, if $X$ and $Y$ differ in exactly one element and their intersection is feasible. This gives rise to the basis graph $G(\Delta)$ of a greedoid $\Delta$ on the set of bases of $\Delta$, whose edges represent pairs of adjacent bases. If $v, u_{1}, u_{2}, \ldots, u_{n}$ are distinct vertices of $G(\Delta)$, then a fan between $v$ and $\left\{u_{1}, u_{2}, \ldots, u_{n}\right\}$, denoted by $v-\left\{u_{1}, u_{2}, \ldots, u_{n}\right\}$, is a set of $n$ paths having only $v$ in common and connecting $v$ to each vertex of $\left\{u_{1}, u_{2}, \ldots, u_{n}\right\}$.

With $D=(V, E, r)$ we denote a finite rooted directed multigraph with vertex set $V$, arc set $E$, and a specified vertex $r \in V$, which we call the root of $D$. We define the in-degree of a vertex $v \in V$ as the number of nonparallel in-arcs of $v$. Let $\mathscr{F}$ be the collection of arc sets of trees of $D$ that contain $r$ and are directed away from $r$ (each vertex $v \neq r$ of such a tree has one arc of the tree coming in). Such trees are called branchings or out-trees. Then $\Delta=(E, \mathscr{F})$ is a greedoid, which we call the branching greedoid (of $D$ ). The minimum degree of $D$, denoted by $\delta(D)$ or $\delta$, is the minimum in-degree taken over all $v \in V \backslash\{r\}$. If every vertex of $D$ is at distance at most 1 from $r$, we say that $D$ is starlike. Two or more (directed) paths are called (internally) disjoint if they have at most their end vertices in common. Let $k$ be an integer and $k \geq 2$. Assuming that $D$ is not starlike and that $D$ admits a spanning out-tree (F्F contains a basis with cardinality $|V|-1$ ) we say that $D$ and, equivalently, $\Delta$ is $k$-connected if no vertex $v$ of $D$ can be separated from $r$ by removing less than $k$ vertices different from $r$ and $v$, or equivalently, if there are $k$ disjoint directed paths from $r$ to any other vertex not adjacent to $r$ by an arc directed away from $r$. If $D$ is starlike, we define the connectivity of $\Delta$ equal to $|V|-1$ (the rank of $\Delta$ ). It is easy to check that this definition coincides with the definition of the connectivity of a general greedoid given in [3, Definition 2.3']. If $X$ is a basis of a branching greedoid $\Delta$ and $v$ is a vertex of the corresponding out-tree with out-degree zero, then $v$ is called a leaf of $X$; if $u v$ is an arc of this out-tree, then $u v$ is called a leaf $\operatorname{arc}$ of $X$. 


\section{RESULTS}

Theorem 1. Let $\Delta$ be a 3-connected branching greedoid of a rooted directed multigraph $D=(V, E, r)$. Then the basis graph $G(\Delta)$ of $\Delta$ is $(\delta-1)$ connected.

To prove Theorem 1 we use the following lemmas, the first of which is [2, Corollary 3.3], and the second of which follows immediately from the definition of $\delta$.

Lemma 2. The basis graph of a 2-connected branching greedoid is connected.

Lemma 3. Let $\Delta$ be a 2-connected branching greedoid of $D=(V, E, r)$ and let $v \in V \backslash\{r\}$. Then at least $\delta$ bases of $\Delta$ contain $v$ as a leaf.

Proof. The branching greedoid $\Delta_{v}$ of $D-v$ is connected and hence has a basis $X$ corresponding to a spanning out-tree of $D-v$. Since there are at least $\delta \operatorname{arcs} e_{1}, e_{2}, \ldots, e_{\delta}$ of $D$ ending at $v, X \cup\left\{e_{1}\right\}, X \cup\left\{e_{2}\right\}, \ldots, X \cup$ $\left\{e_{\delta}\right\}$ are $\delta$ bases of $\Delta$ that all contain $v$ as a leaf.

Lemma 4. Let $\Delta$ be a 3-connected branching greedoid of $D=(V, E, r)$ and let $v \in V \backslash\{r\}$. Then the set $B_{v}:=\{X \mid X$ is a basis of $\Delta$ with leaf $v\}$ induces a $(\delta-1)$-connected subgraph of $G(\Delta)$.

Proof. Let $e_{1}, e_{2}, \ldots, e_{k}$ be the arcs of $D$ ending at $v$ and starting at $\boldsymbol{v}_{1}, \boldsymbol{v}_{2}, \ldots, \boldsymbol{v}_{k}$. Clearly $k \geq \boldsymbol{\delta}$ and the branching greedoid $\Delta_{v}$ of $D-\boldsymbol{v}$ is 2-connected. By Lemma 2, $G\left(\Delta_{v}\right)$ is connected. Now the following observations are obvious.

(4) For any basis $X$ of $G\left(\Delta_{v}\right)$, the set of bases $\left\{X \cup\left\{e_{1}\right\}, X \cup\left\{e_{2}\right\}, \ldots\right.$, $\left.X \cup\left\{e_{k}\right\}\right\} \subseteq B_{v}$ induces a complete graph in $G(\Delta)$.

(5) If, for two bases $X$ and $Y$ of $G\left(\Delta_{v}\right)$, the edge $X Y$ corresponds to an exchange of two leaf arcs ending at a common leaf $w$ of $X$ and $Y$, then $X \cup\left\{e_{j}\right\}$ is adjacent to $Y \cup\left\{e_{j}\right\}$ in $G(\Delta)$ whenever $w \neq v_{j}(j=$ $1, \ldots, k)$.

Hence the subgraph $H$ of $G(\Delta)$ induced by $B_{v}$ arises from $G\left(\Delta_{v}\right)$ by replacing each vertex by a $k$-clique, and each edge by $k-1$ independent edges according to (4) and (5). It is clear that, for any set $S \subseteq V(H)$ with $|S|<$ $\delta-1 \leq k-1, H-S$ is connected. Hence $H$ is $(\delta-1)$-connected.

Lemma 5. Let $D=(V, E, r)$ be 3-connected and let $X$ be a basis of the branching greedoid $\Delta$ of $D$. Then for any $v \in V \backslash\{r\}$ there are $\delta-1$ bases $X_{1}, X_{2}, \ldots, X_{\delta-1}$ of $\Delta$ such that $X_{i}(i=1,2, \ldots, \delta-1)$ contains $v$ as a leaf and there is a fan $X-\left\{X_{1}, X_{2}, \ldots, X_{\delta-1}\right\}$ in $G(\Delta)$. 
Proof. We distinguish two cases.

Case 1. $v$ is a leaf of $X$.

Let $e$ be the leaf arc of $X$ ending at $v$. There are at least $\delta-1$ arcs $e_{1}, e_{2}, \ldots, \mathrm{e}_{\delta-1}$ ending at $v$ and different from $e$. Now $X_{i}=\left(X \cup\left\{e_{i}\right\}\right) \backslash\{e\}$ $(i=1,2, \ldots, \delta-1)$ is a basis of $\Delta$ containing $v$ as a leaf, and $X$ is adjacent to $X_{i}(i=1,2, \ldots, \delta-1)$. Hence there is a fan $X-\left\{X_{1}, X_{2}, \ldots, X_{\delta-1}\right\}$.

Case 2. $v$ is not a leaf of $X$.

Let $e$ be a leaf arc of $X$ ending at $u$. Consider the branching greedoid $\Delta_{u}$ of $D-u$. Clearly, $\Delta_{u}$ is 2-connected, and $X \backslash\{e\}$ is a basis of $\Delta_{u}$. Since $v$ is a vertex of $D-u$, by Lemma 3 there are at least $\delta-1$ bases $X_{1}^{\prime}$, $X_{2}^{\prime}, \ldots, X_{\delta-1}^{\prime}$ of $\Delta_{u}$ with common leaf $v$. Let $X_{i}:=X_{i}^{\prime} \cup\{f\}$ for some $\operatorname{arc} f$ ending at $u(i=1,2, \ldots, \delta-1)$. By Lemma $4, X, X_{1}, X_{2}, \ldots, X_{\delta-1}$ are vertices of a $(\delta-1)$-connected subgraph of $G(\Delta)$, implying that $G(\Delta)$ contains a fan $X-\left\{X_{1}, X_{2}, \ldots, X_{\delta-1}\right)$.

Proof of Theorem 1. Let $X$ and $Y$ be two bases of $\Delta$. It is sufficient to prove that there are at least $\delta-1$ disjoint paths in $G(\Delta)$ connecting $X$ and $Y$.

Denote by $L(X)$ and $L(Y)$ the set of leafs of $X$ and $Y$, respectively.

If $L(X) \cap L(Y) \neq \varnothing$, the result follows from Lemma 4 .

Suppose $L(X) \cap L(Y)=\varnothing$ and let $v \in L(Y)$. By Lemma 5, there is a fan $X-\left(X_{1}, X_{2}, \ldots, X_{\delta-1}\right)$ in $G(\Delta)$ such that $v$ is a leaf of $X_{i}$ ( $i=$ $1,2, \ldots, \delta-1)$.

By Lemma 4, there are $\delta-1$ disjoint paths from $X_{i}$ to $Y(i=1$, $2, \ldots, \delta-1)$.

Letting $i$ run through $1,2, \ldots, \delta-1$, we obtain a collection of paths connecting $X$ and $Y$ from which we can choose $\delta-1$ disjoint paths connecting $X$ and $Y$. This can be shown as follows. If we delete a set of $t \leq \delta-2$ vertices from $G(\Delta)$ not containing $X$ or $Y$, then at least one path connecting $X$ and $X_{i}$ remains and at least one of the paths connecting $X_{i}$ and $Y$ remains.

This completes the proof of Theorem 1 .

The following result is an immediate consequence of Theorem 1.

Corollary 6. Let $\Delta$ be a 3-connected branching greedoid and suppose, for some $n_{1}, n_{2} \in \mathbb{N}$ with $n_{1}<n_{2}$, there are bases $X_{1}$ and $X_{2}$ of $\Delta$ with $n_{1}$ and $n_{2}$ leaves, respectively. Then, for any $n \in \mathbb{N}$ with $n_{1}<n<n_{2}$, there are at least $\delta-1$ bases of $\Delta$ with $n$ leaves.

If $X_{1}$ and $X_{2}$ in Corollary 6 have a common leaf $v$, then all $\delta-1$ bases can be chosen to have $v$ as a leaf. This follows from Lemma 4 . 


\section{CONCLUDING REMARKS}

Let $\mathscr{H}$ be the class of strongly $\delta$-connected directed graphs that contain a directed Hamilton path (a directed path containing all vertices of the graph). Let $\mathscr{G}$ be the class of rooted directed graphs obtained from members of $\mathscr{H}$ in the following way. To every $H \in \mathscr{H}$ add a root $r$ and another new vertex $v$. Also add arcs from $r$ to all vertices of $H$, and arcs ending at $v$ and starting from precisely $\delta$ vertices of $H$ including the end vertex of a directed Hamilton path of $H$.

Let $G \in \mathscr{G}$ be obtained from $\mathrm{H} \in \mathscr{H}$ in this way. It is clear that $G$ is nonstarlike with $\delta(G)=\delta$ and that $G$ contains a directed Hamilton path $P$ from $r$ to $v$. Let $X$ be the set of arcs of $P$. Then $X$ is a basis of the branching greedoid $\Delta$ of $G$ and there are precisely $\delta-1$ bases of $\Delta$ adjacent to $X$ (they only differ in the arc ending at $v$ ). Hence the basis graph $G(\Delta)$ of $\Delta$ is (at most) $(\delta-1)$-connected, showing that we cannot replace " $(\delta-1)$ connected" in Theorem 1 by " $t$-connected" for $t \geq \delta$. In this sense, Theorem 1 is best possible.

If $r$ is a unique cut vertex of $D=(V, E, r)$, we can do better by considering the subgraphs of $D$ induced by $r$ and the vertices of each of the components of $D-r$. Let $D_{1}, D_{2}, \ldots, D_{m}$ denote these subgraphs. Then it is easy to see that $G(\Delta)$ is isomorphic to the (Cartesian) product graph of $G\left(\Delta_{1}\right)$, $G\left(\Delta_{2}\right), \ldots, G\left(\Delta_{m}\right)$, where $\Delta, \Delta_{1}, \ldots, \Delta_{m}$ are the branching greedoids of $D$, $D_{1}, \ldots, D_{m}$, respectively. This implies that $G(\Delta)$ is at least $\left(k_{1}+k_{2}+\cdots+\right.$ $\left.k_{m}\right)$-connected if $G\left(\Delta_{i}\right)$ is at least $k_{i}$-connected $(i=1,2, \ldots, m)$.

Finally, let $v$ be a vertex of $D$ at distance 1 from the root $r$. If $v$ has only one in-arc, we can contract this arc $(r, v)$ to obtain a new digraph $D^{\prime}$. It is easy to see that the basis graph of the branching greedoid of $D$ is isomorphic to that of $D^{\prime}$. Therefore, we may always assume that every vertex of $D$ at distance 1 from the root $r$ has at least two in-arcs.

\section{ACKNOWLEDGMENT}

We would like to thank Professor U. Faigle for bringing greedoids to our attention.

\section{References}

[1] A. Björner and G. M. Ziegler, Introduction to greedoids. Combinatorial Geometries: Advanced Theory, Cambridge University, preprint (1987).

[2] B. Korte and L. Lovász, Basis graphs of greedoids and two-connectivity. Math. Program. Study 24 (1985) 158-165.

[3] G.M. Ziegler, Branchings in rooted graphs and the diameter of greedoids. Combinatorica 8 (1988) 217-234. 